and binds rhodopsin, the $\beta$ and $\gamma$ subunits, and CGMP-PDE. Diffraction quality crystals of the heterotrimeric complex are grown in microseeded hanging drops containing $10 \mathrm{mg} / \mathrm{ml}$ protein, $10 \%$ PEG-8000, $50 \mathrm{mM}$ Tris, pH $8.0,50 \mathrm{mM} \mathrm{NaCl}, 10 \%$ glycerol, and $0.1 \% \beta$-mercaptoethanol. The space group is $C 2$ with unit cell dimensions of $a=133.4, b=91.4, c=83.2, \beta=120.1^{\circ}$. Progress to date will be reported.

PSO4.10.22 REFINED CRYSTAL STRUCTURES OF TNFALPHA AIND TINT IMUTANT R31D. C. Reed", Z.-Q. Fu*, J. Wu*, Y.-N. Xue, M.-J. Chen and I.T. Weber* *Department of Pharmacology, Department of Microbiology and Immunology, Jefferson Cancer Center, Thomas Jefferson University. Philadelphia PA 19107, USA.

Crystal structures have been determined of recombinant human tumor necrosis factor- $\alpha(\mathrm{TNF}-\alpha)$ and its R31D mutant that preferentially binds to TNF receptor $\mathrm{R} 1$ with five times greater affinity than to receptor $\mathrm{R} 2$. Crystals of the wild type TNF were of space group $P_{1} 2_{1} 2$ and had unit cell dimensions of $a=b=94.7$ and $c=117.4 \mathrm{~A}$. Refinement of the structure gave an R-factor of $22.3 \%$ at $2.5 \AA$ resolution. The crystals of TNF R31D mutant diffracted to $2.3 \AA$ resolution, and were of identical space group to the wild type with unit cell dimensions of $a=b=95.4$ and $c=116.2$ $\AA$, and the structure was refined to an R-factor of $21.8 \%$. Almost continuous electron density was observed throughout both structures, although the first five residues of the $\mathrm{N}$-termini appear to be disordered. Comparison of the structures of the wild type and mutant TNF showed that the two trimers were similar with an rms deviation of $0.77 \AA$ for main chain atoms, however, the subunits within each trimer were more variable with mus deviations of over $1.05 \AA$ for pairwise comparison of main chain atoms. Model complexes of TNF with receptors $R 1$ and $R 2$ have been used to predict TNF-receptor interactions. The Arg 31 of wild type TNF is predicted to form an ionic interaction with an identical glutamic acid in both receptors $\mathrm{R} 1$ and $\mathrm{R} 2$. In the TNF R31D mutant, modeling suggested that this interaction is replaced by interaction with a histidine in R1, but there is no equivalent interaction in R2, consistent with the observed greater affinity of the R31D mutant for receptor $R 1$ compared to $R 2$.

PS04.10.23 REEINEMENT OF NATIVE AND MUTANT VEROTOXN B-SUBUNIT STRUCTURES. Allan M. Sharp, Penelope E. Stein, Amechand Boodhoo, and Randy J. Read. Departments of Biochemistry, and Medical Microbiology and Immunology, University of Alberta, Edmonton, Alberta, Canada, T6G 2H7

The ability of enterohemorrhagic $E$. coli strains to cause hemorrhagic colitis and henolytic uremic syndrome is a result of the production of a shiga-like toxin or verotoxin. This is an $A B_{5}$ type toxin, with a catalytic $A$-subunit attached to a pentamer of $B$ subunits that bind the cell surface glycolipid globotriaosylceramide $(G b-3)$. The solution of the wild-type verotoxin $B$-subunit pentamer, at $2.2 \AA$ resolution, revealed a predominantly $\beta$-sheet structure, built around a central helix-lined pore [1]. Based on the distribution of conserved surface residues, the deep clefts at the interfaces between the subunits were proposed to be the sugarbinding sites. Mutation of a phenylalanine to an alanine residue in the cleft region (Mutant F30A) did eliminate the majority of glycoside binding [2]. Difference Fourier analysis of crystals of F30A at $2.0 \AA$ resolution suggested that there were no major structural differences in the protein away from the mutation site. Further refinement of the native and mutant structures with XPLOR and TNT has reduced their R-factors to 0.195 and has allowed detailed analyses of the features of the structures, their distortion from ideal pentamers by crystal contacts, changes at their putative binding sites, and changes in occupancy at two crystallographic zinc binding sites.

A more recent $2.8 \AA$ crystal structure of the verotoxin Bsubunit complexed with $\mathrm{Gb} 3$ has shown that there are in fact three different classes of binding sites on the pentamer surface, one of them closely corresponding with the original prediction, and another contacting phenylalanine 30 [3]. The effects of the mutation may aid in elucidating the relative importance of the different sites.

Research supported by the AHMRF, MRC. HHMI.

[1] Stein, P. E. et al., Nature, 355, 748-750(1992).

[2] Clark, C. et al. Molecular Microbiology, in press.

[3] Ling, H. et al, W127, p.179, ACA Annual Meeting, Montreal, 1995.

PS04.10.24 ATOMIC STRUCTURE OF POTD-THE PRIMARY RECEPTOR OF SPERMIDINE/PUTRESCINE TRANSPORT SYSTEM IN E.COLI. D.G.Vassylyev], S. Sugiyama2, M. Matsushima3, K. Kashiwagi4, K. Igarashi4, K. Morikawal, 1 Biomolecular Engineering Research Institute, 6-2-3, Furuedai, Suita, Osaka, 565, Japan, 2Kyowa Hakko Kogyo Co. Ltd., Pharmaceutical Research Laboratories, 1188 Shimotogari, Nagaizumi-cho,Sunto-gun,Shizuoka,411,Japan, 3Rational Drug Desighn Laboratories, 4-1-1 Misato, Matsukawa, Fukushina 96012, Japan, 4Faculty of Phamaceutical Sciences, Chiba Univ., 133 Yayoi-cho, Inage-ku, Chiba 263. Japan

The crystal structure of PotD (a periplasmic binding protein which is the primary receptor of polyamine transport system in E.coli) in complex with spermidine has been solved at $2.5 \AA$ resolution. The PotD protein ( 325 amino acids) consists of two domains with a deep cleft ( $20 \AA$ long, $5 \AA$ wide, $14 \AA$ deep) in the interface between them. This cleft was found to be a binding site of spermidine in the complex. The three positively charged nitrogens of spermidine are recognized by four acidic side chains of PotD in the cleft while five aromatic residues anchor the spermidine methylene backbone by van der Waals interactions. The overall fold of PotD is similar to other periplasmic binding proteins despite the fact of low sequence similarity.

Crystals of the complex belong to the space group $\mathrm{P} 2{ }_{1}(\mathrm{a}=145.3 \AA, b=69.1 \AA, c=72.5 \AA, \beta=107.6, Z=8)$. The structure was solved by MIR method in combination with solvent flattening and 4 -fold N.C.S. averaging and refined at $2.5 \AA$ resolution to a final $\mathrm{R}$-factor of 0.199 (R-free $=0.280$ ).

PS04.10.25 CRYSTALLOGRAPHIC ANALXSIS OF THE MAP KINASE P38. Zhulun Wang1, Jiahuai Han2 \& Elizabeth J. Goldsmith 1, Department of Biochemistry, UT Southwestern Medical Center at Dallas, Dallas, TX 75235i, Department of Immunology, The Scripps Research Institute, La Jolla, CA 920372

The mitogen-activated protein (MAP) kinase cascades are major signaling pathways that transmit extracellular information from the cell surface into the intracellular responses. Various extracellular stimuli, such as, growth factors, heat, UV-irradiation, inflammatory cytokines and hyperosmolarity, activate MAP kineses by dual phosphorylation on threonine $(T)$ and tyrosine $(Y)$. Three distinct MAP kineses signal transduction pathways have been defined so far in mammalian cells based on their differential activation selectivity and substrate specificity. As a new member of the MAP kinase family, p38 has been identified in a stress-activated signal transduction pathway. The three dimensional structure of p38 and comparison of p38 to ERK2 will help to elucidate the mechanisms of specificity determination in this family.

His6-tagged recombinant murine p38 $(45 \mathrm{kDa})$ protein has been expressed, purlfied and crystallized by vapor diffusion method 
in hanging-drop with $20 \%$ PEG8000 as precipitant. The typical crystal reaches $0.6 \times 0.3 \times 0.06 \mathrm{~mm}$ in two weeks. The crystals belong to the orthorhombic space group $\mathrm{P}_{2} 2_{2} 2_{1}$ with unit cell dimensions of $a=46.1 \AA, b=86.9 \AA$ and $c=126.5 \AA$. Native clatasets, with diffraction limit of $2.2 \AA$, at both room temperature and low temperature, have been collected on a Rigaku it-axis. Molecular replacement using the MAP kinase ERK2 as a model structure and multiple isonorphous replacement have been applied to solve the structure.

PS04.10.26 $1.7 \AA$ A STRUCTURE OF THE C-TERMINAL SH2 DOMAIN OF THE PS5 SUBUNTT OF HUMAN PHOSPHATD YLINOSITOL 3-KINASE. Simon Weston, Dean Derbyshire, Alex Breeze \& Richard Pauput, Zeneca Pharmaceuticals, Alderley Park, Macclesfield, UK

The C-terminal SH2 domain of the $85 \mathrm{kDa}$ regulatory subunit of human phosphatidylinositol 3-kinase (an enzyme responsible for a key mitogenic signal invoked by a variety of growth factors) complexed with a specific high-affinity phosphorylated tyrosine pentapeptide sequence from the platelet-derived growth factor receptor has been crystallised in spacegroup C2 and diffracts to $1.7 \AA$.

All molecular replacement efforts using other SH2 structures as trial models had failed - the structure could only be solved once the NMR structure carried out in our laboratory had produced a sufficiently accurate trial model. This was probably because the crystals were densely packed and the molecular replacement signal suffered from interference from cross-vectors. The structure is currently being refined and will be presented and compared to other SH2 structures.

\section{PS04.10.27 CRYSTAL STRUCTURE OF HUMAN} INTERLEUKIN-10 AT $1.6 \AA$ RESOLUTION. Alexander Zdanov, Celine Schalk-Hihi, Alexander Wlodawer, Macromolecular Structure Laboratory, NCI-Frederick Cancer Research and Development Center, ABL-Basic Research and Development Center, ABL-Basic Research Program, Frederick, Maryland, 21702

Interleukin-10 (IL-10) is a cytokine that inhibits production of a number of regulatory factors. The molecule is a homodimer of two intepenetrating monomers ( 160 amino acid residues each), forming a V-shaped structure. Each half of the structure consists of six $\alpha$-helices. four originating from one monomer and two from the other. The overal topology of the IL-10 bears close resemblance to interferon $\gamma$.

The crystal structure of human interleukin-10 (IL-10) has been refined at $1.6 \AA$ resolution against X-ray diffraction data collected at $100 \mathrm{~K}$ with the use of synchrotron radiation. Although similar to the IL-10 structure determined previously at room temperature, the low-temperature structure contains, in addition, four Nterminal residues, three sulfate anions, and 175 extra water molecules. Whereas the main-chain conformation is preserved, about $30 \%$ of the side chains, most of them on the protein surface, assume different conformations, and the protein itself appears to contract slightly at low temperature.

A computer model of a complex of IL-10 with its two soluble domains of its receptor has been generated based on the topological similarity of $I L-10$ to interferon- $\gamma$. The contact region between the IL-10 and each receptor shows excellent complementarity of polar and hydrophobic interactions, suggesting that the model is generally correct.
PR04.10.28 COMBINED STUDY OR PURINERGIC TRANSDUCTION USING CRYSTALLOGRAPIC AND ELECTROPHYSIOLOGICAL TECHNIQUES V. A. Panchenko, F. Rodrigues-Pascuali", M. T. Miras-Portugal", O.A.Krishtal, A. A. Bogomoletz Institute of Physiology, Kiev, Ukraine, "Departamento de Bioquimica, Facultad de Veterinaria, Universidad Complutense Madrid, Spain.

The purinergic transduction in manmalian brain is not conpletely investigated by this monent. The aim of this study was to compare the space distribution of diadenosine polyphosphates binding sites in rat brain by the means of autoradiography with possible contribution of this compounds in synaptic transmission mechanisms studied electrophysiologicaly. The distribution of the diadenosine tetraphosphate $(\mathrm{Ap} 4 \mathrm{~A})$ high affinity binding sites has been studied in rat brain by autoradiographic method using $[3 \mathrm{H}]-\mathrm{Ap} 4 \mathrm{~A}$ as the ligand. Due to specific activity of the labelled ligand, all of the tissues binding experiments were carried out to $1 \mathrm{nM}$ concentration. The Ap4A binding sites were widespread but heterogeneously distributed throe the rat brain. The range of densities was from 3 to 15 fmol/mg tissue in grey matter areas, while white matter was devoid of specific binding, showing background levels. In electrophysiological study the action of Ap4A on potential-dependent Ca channels was studied. Ap4A was found to cause a potentiation of $\mathrm{N}$-type Cat+ channels by $21 \pm 6 \%(n=12)$. This potentiation was observed in hippocampal CA3 neurons but not in cerebellar Purkinje neurons. The pharmacology of such potentiation was typical for $\mathrm{N}$-channels properties i.e. it was blocked by 5 micromolar of w-Conotoxin but not 50 micromolar nimodipine. It was possible to compare the data obtained by these two techniques and make a correlation in space distribution and functional properties of Ap4A receptors. In conclusion, our results support the existence of Ap4A specific binding sites and their contribution into Ca-regulated synaptic mechanisms.

\section{Protein Carbohydrate Structures}

MS04.11.01 ATOMLC INTERACTIONS BETWEEN PROTEINS AND CARBOHYDRATES. F. A. Quiocho" N. K. Vyas", M. N. Vyas" J. C. Spurlino*, L. E. Rodseth"* and D. R. Bundlet. "Howard Hughes Medical Institute and Department of Biochemistry, Baylor College of Medicine Houston, Texas 77030 , USA and TDepartment of Chemistry, University of Alberta, Edmonton, Alberta TGG 2C6. Canada

Major features of the atomic interactions between proteins and carbohydrates that will be presented are based on the following refined structures: i) L-arabinose-binding protein (ABP) complexed with L-arabinose, D-galactose, D-fucose, and 4 deoxy and halide derivatives of $\mathrm{D}$-galactose; ii) $\mathrm{D}$-glucose/D-galactose-binding protein (GGBP) complexed with D-glucose and D-galactose; iii) maltodextrin-binding protein (MBP) complexed with maltose, maltotriose, maltotetraose and $B$-cyclodextrin; and iv) antibody against the lipolysaccharides $\mathrm{O}$-antigen of Shigella flexneri in the absence and presence of trisaccharide and pentasaccharide $O$-antigen segments. The first three proteins serve as initial receptors for bacterial active transport and chemotaxis. All proteins that bind carbohydrates can be distinguished into two groups or types depending primarily on the location of the binding site. Whereas the sites in proteins belonging to Group I (e.g., ABP, GGBP, lacl family of repressors) are buried and able to sequester the ligands, those of group II (e.g, immunoglobulins, lectins, etc.) are near the protein surface. Each group further possesses somewhat unique features. $\mathrm{MBP}$ has a binding site groove which exhibits properties of the two groups. Features of protein-carbohydrate interactions that will be discussed include i) hydrogen-bonding interactions, ii) van der Waals interactions, especially those arising from stacking interactions with aromatic residues, iii) role of water molecule, iv) structural plasticity, v) bound oligosaccharide confomations, vi) specificity, and vii) affinity. 